Vol 12, Issue 2, 2019

\title{
PROTECTIVE EFFECT OF ZILEUTON AND MK-866 AGAINST HEPATIC DAMAGE INDUCED BY DOXORUBICIN
}

\author{
HUSSAM H SAHIB, AHMED M SULTAN*, HUSSEIN A SAHEB \\ College of Pharmacy, University of Al-Qadisiyah, Iraq. Email: surprisor82@yahoo.com
}

Received: 19 November 2018, Revised and Accepted: 02 January 2019

\begin{abstract}
Objective: The present study was designed to investigate the protective effect of Zileuton and MK-886 against hepatic damage induced by doxorubicin.

Methods: A total of 30 healthy adult male albino rats were randomized and rats were divided into five groups, six animals in each: Control negative group, Vehicle group: Rats were given ethanol i.p., Dx group: Doxorubicin (15 mg/kg), Mk group: Mk-886-treated rats given 0.6 mg/kg of Mk-886 i.p, and Z group: Zileuton-treated rats given zileuton $10 \mathrm{mg} / \mathrm{kg}$ i.p. Biochemical tests of the serum for ASAT and ALAT level were estimated. Serum glutathione $(\mathrm{GSH})$ concentrations $(\mu \mathrm{g} / \mathrm{ml}$ ) were determined using GSH ELISA Kit, while serum malondialdehyde (MDA) concentrations (ng/ml) were determined using MDA ELISA Kit. Livers were removed from each rat and fixed in 10\% neutral-buffered formalin and embedded in paraffin for histopathological studies.
\end{abstract}

Results: MK- and zileuton-treated groups showed higher GSH levels and lower MDA levels as compared with Dx-treated group. MK-886 associated with significant $\mathrm{p}<0.05$ decreased the liver enzymes in comparison with doxorubicin-treated rats. Zileuton showed insignificant ( $\mathrm{p}>0.05$ ) changes. The liver tissues that treated with Dx only showed several histopathological changes such as moderate sinusoidal dilation, vacuolation, mild-tomoderate hepatocyte necrosis/degeneration and inflammatory cell infiltration, and severe congestion. Liver tissues that treated by zileuton with Dx showed sinusoidal dilation, vacuolation, mild congestion, and inflammatory cell infiltration, while those treated with Mk-886 plus Dx showed nearly normal liver pathophysiology.

Conclusion: It has been concluded that Zileuton and MK-886 have protective effects against hepatic damage induced by doxorubicin.

Keywords: Zileuton, Doxorubicin, MK-886, Hepatic damage.

(C) 2019 The Authors. Published by Innovare Academic Sciences Pvt Ltd. This is an open access article under the CC BY license (http://creativecommons. org/licenses/by/4. 0/) DOI: http://dx.doi.org/10.22159/ajpcr.2019.v12i2.30815

\section{INTRODUCTION}

Doxorubicin is a potent anthracycline anticancer drug used to treat different types of malignancies [1] such as breast cancer, leukemia, and AIDS-related Kaposi's sarcoma [2,3]. Further, it is also used in the treatment of cancers of bladder, testes, ovaries, lung, cervix, prostate, and Ewing's sarcoma [4]. The clinical effectiveness of DXR in the management of a wide range of malignant diseases is influenced by various dose-limiting adverse effects such as hepatotoxicity, nephrotoxicity, and cardiomyopathy [5-7]. Systemic toxicity is occurring clinically at doses less than the therapeutic doses [8]. The toxicity is started at imbalance the free radical production by two pathways: Enzymatic pathway using a respiratory chain of the mitochondrial and a non-enzymatic pathway occurred by utilizing iron [9]. The apoptotic processes are increasing in hepatic tissues after taking one dose of DOX [10]. The DOX dose increased lipid peroxidation in mitochondria and microsomes in the liver tissue, particularly with iron ions [11]. The DOX causes hepatotoxicity such as steatosis, vascular damage, and focal damage in hepatocytes [12].

Leukotrienes (LT) considered as a pro-inflammatory lipid mediator that is produced by the action of 5-lipoxygenase enzyme on arachidonic acid [13]. Zileuton is an inhibitor of 5-lipoxygenase activity if takes it orally and so that zileuton can block all products of the 5-lipoxygenase (CysLTs and LTB4). It is used to ameliorate the symptoms of asthma $[14,15]$. MK-886 (investigational compound) is an inhibitor of leukotriene production in vitro and in vivos. Moreover, this agent inhibits the activation and translocation of 5-lipoxygenase by inhibiting leukotriene biosynthesis and by an indirect pathway through the binding of a membrane-bound 5-lipoxygenase-activating protein (FLAP) [16].

\section{MATERIALS AND METHODS}

Animals

A 30 healthy adult male albino rats with average weight $(230 \pm 30 \mathrm{~g})$ were housed in animal's house of the College of Pharmacy, University of Al-Qadisiyah, Iraq. Under $12 \mathrm{hr}$. light/12 hr. dark at 25C, rats were fed. Rats were fed standard rodent chew diet and tap water. This study continued for 3 days. Protocol of the current research was approved by the Institute Review Board at the College of Pharmacy, University of Al-Qadisiyah, Iraq.

\section{Materials}

Doxorubicin was used (50 mg in $25 \mathrm{ml}$, EBEWE Pharma Ges. m.b.H., 4866-A Unterach., Austria.), MK-886 powder was from Santa Cruz Biotechnology, Inc. (Lot H3116), and zileuton powder was from Santa Cruz Biotechnology, Inc. (Lot A2513).

\section{Experimental design}

Rats were divided into five groups, six animals in each:

- Control negative group: Rats take standard diet and water.

- Vehicle group: Rats were given ethanol i.p.

- Dx group: Rats given doxorubicin (15 mg/kg intraperitoneally) single dose daily [17].

- Mk group: Mk-886-treated rats given $0.6 \mathrm{mg} / \mathrm{kg}$ of Mk-886 i.p $30 \mathrm{~min}$ before doxorubicin administration [16].

- Z group: Zileuton-treated rats given zileuton $10 \mathrm{mg} / \mathrm{kg}$ i.p $30 \mathrm{~min}$ before doxorubicin administration [18].

After 3 days, all rats were anesthetized by intraperitoneal injection of thiopental sodium $(70 \mathrm{mg} / \mathrm{kg})[19]$. 
Assay of serum ALAT and ASAT concentrations

Blood was drawn and quickly put in gel separator tubes to clot at $25^{\circ} \mathrm{C}$. The samples were centrifuged for $10 \mathrm{~min}$ at $3000 \mathrm{rpm}\left(30^{\circ} \mathrm{C}\right)$. The supernatant layer of the serum appears and transfers to another clean tube. Then, samples were frozen at $-30^{\circ} \mathrm{C}$ for long-term preservation for making biochemical tests of the serum for the estimation of ASAT and ALAT level.

Assay of glutathione (GSH) and malondialdehyde

(MDA) concentrations

Serum GSH concentrations ( $\mu \mathrm{g} / \mathrm{ml}$ ) were determined using GSH ELISA Kit Catalog No: E-EL-0026 (96T) that obtained from Elabscience Biotechnology Inc., while serum MDA concentrations (ng/ml) were determined using MDA ELISA Kit Catalog No: E-EL-0060 (96T) that obtained from Elabscience Biotechnology Inc. Both GSH and MDA assay procedures were done according to the procedures that mentioned in the kit leaflet.

\section{Histopathological examination of the liver}

Livers were removed from each rat and fixed in $10 \%$ neutral buffered formalin and embedded in paraffin for histopathological studies.

The histopathological changes divided into several grades as follows:

1. - (normal)

2. + (mild).

3. ++ (moderate)

4. +++ (severe) $[20]$

\section{Statistical analysis}

The samples data were submitted to the analysis by SPSS program 12.0 edition using one-way analysis of variance and then submitted for Dunnett's test at $\mathrm{p}<0.05$.

\section{RESULTS}

Effect of zileuton and MK-886 treatment on liver function biomarkers

As shown in Fig. 1, doxorubicin caused a significant $(\mathrm{p}<0.05)$ increased serum liver enzyme (ASAT and ALAT) concentration when compared with negative control group. MK-886 associated with significant $\mathrm{p}<0.05$ decreased the liver enzymes in comparison with doxorubicin-treated rats. Zileuton showed insignificant $(p>0.05)$ changes while vehicle group did not affect study parameters.

\section{Effect of zileuton and MK-886 treatment on GSH and MDA levels}

As shown in Fig. 2 negative control and vehicle groups presented with normal GSH and MDA values, a doxorubicin-treated group associated with significant $(\mathrm{p}<0.05)$ decreases GSH levels and increases MDA levels when compared with negative control group. MK- and zileutontreated groups showed higher $(\mathrm{p}<0.05)$ GSH levels and lower $(\mathrm{p}<0.05)$ MDA levels as compared with Dx-treated group.

\section{Zileuton and MK-886 treatments on liver histopathology}

Table 1 shows the control and vehicle groups in normal liver architecture (Fig. 3a and b). The liver tissues that treated with Dx only showed several histopathological changes such as moderate sinusoidal dilation, vacuolation, mild-to-moderate hepatocyte necrosis/degeneration and inflammatory cell infiltration $(n=4)$, and severe congestion (Fig. 3c-f). Liver tissues that treated by zileuton with Dx showed sinusoidal dilation, vacuolation, mild congestion, and inflammatory cell infiltration as shown in Fig. 3g, while those treated with Mk-886 plus Dx showed nearly normal liver pathophysiology (Fig. 3h).
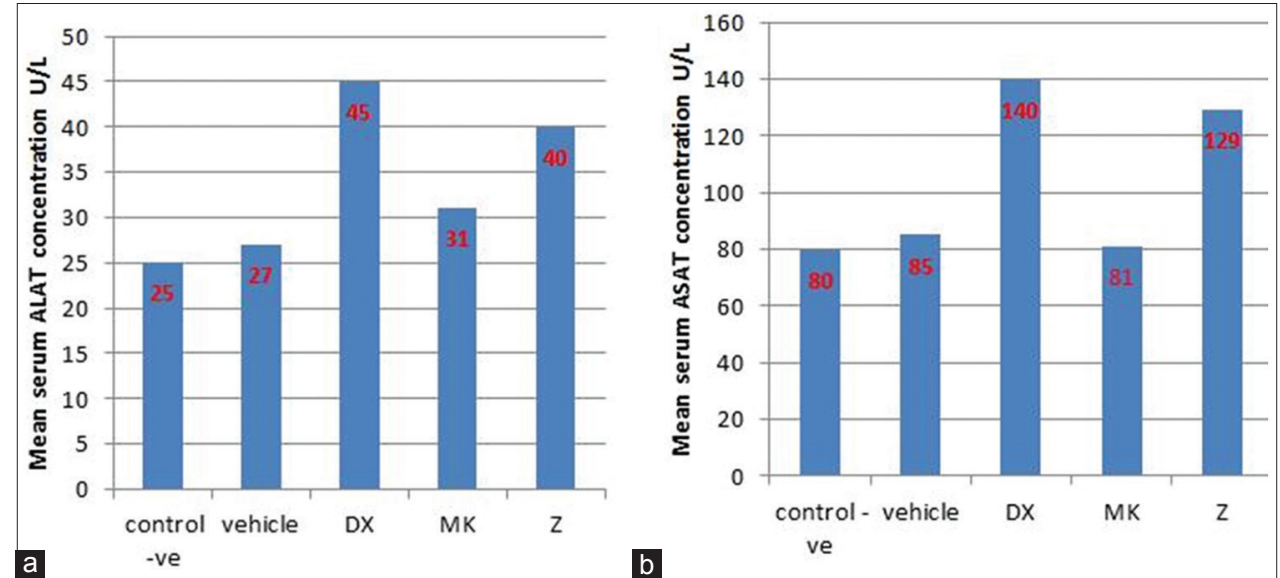

Fig. 1: Liver function test. (a) Mean serum ALAT concentration U/L (b) Mean serum ASAT concentration U/L
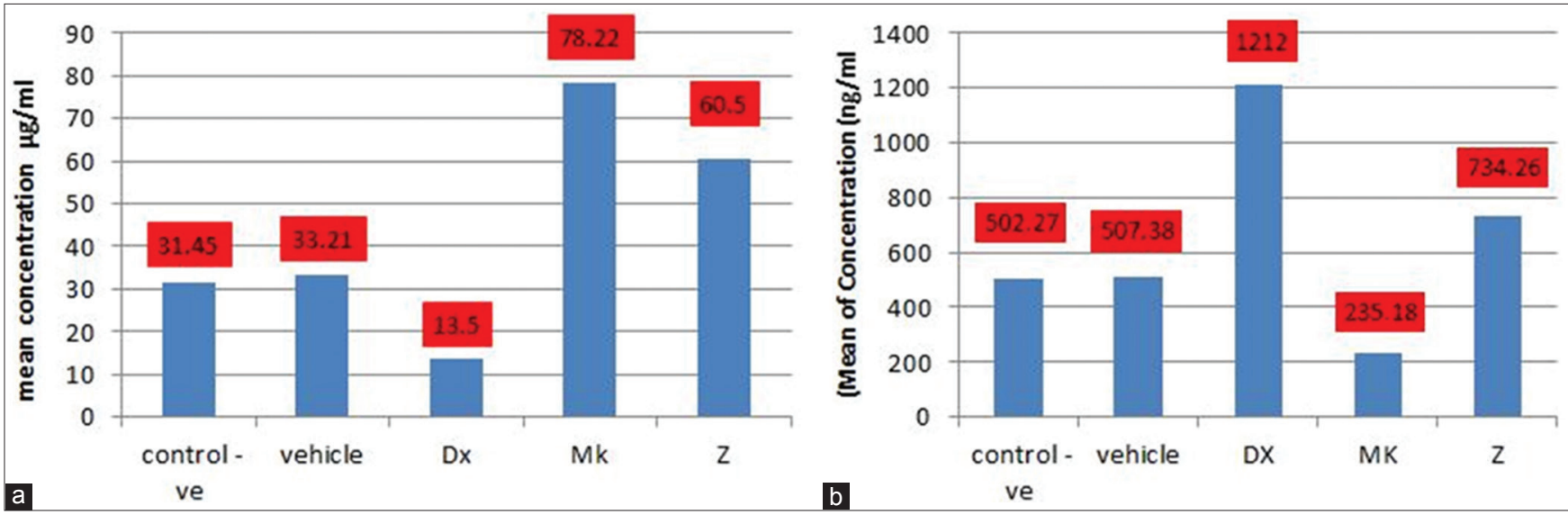

Fig. 2: Oxidative markers in rats. (a) Rat GSH ELISA means concentration in $\mu \mathrm{g} / \mathrm{ml}$, (b) Rat MDA ELISA mean concentration in ng/ml 
Table 1: Histopathological findings in the liver of rats

\begin{tabular}{|c|c|c|c|c|c|}
\hline Findings & Negative control group & Vehicle group & Dx-treated group & Z plus Dx-treated group & Mk plus Dx-treated group \\
\hline Hepatocyte & 0 & 0 & $0(2 / 6)$ & $0(4 / 6)$ & $0(5 / 6)$ \\
\hline \multirow[t]{2}{*}{ degeneration/necrosis } & & & $+1(3 / 6)$ & $+1(2 / 6)$ & $+1(1 / 6)$ \\
\hline & & & $+2(1 / 6)$ & & \\
\hline Inflammatory cell & 0 & 0 & $+1(3 / 6)$ & $0(3 / 6)$ & $0(4 / 6)$ \\
\hline infiltration & & & $+2(3 / 6)$ & $+1(3 / 6)$ & $+1(2 / 6)$ \\
\hline \multirow[t]{3}{*}{ Vacuolation } & 0 & 0 & $0(1 / 6)$ & $0(4 / 6)$ & $0(4 / 6)$ \\
\hline & & & $+1(2 / 6)$ & $+1(2 / 6)$ & $+1(2 / 6)$ \\
\hline & & & $+2(3 / 6)$ & & \\
\hline \multirow[t]{2}{*}{ Sinusoidal dilation } & 0 & 0 & $+1(1 / 6)$ & $0(5 / 6)$ & $0(5 / 6)$ \\
\hline & & & $+2(5 / 6)$ & $+1(1 / 6)$ & $+1(1 / 6)$ \\
\hline \multirow[t]{2}{*}{ Congestion } & 0 & 0 & $+3(6 / 6)$ & $0(3 / 6)$ & $0(5 / 6)$ \\
\hline & & & & $+1(3 / 6)$ & $+1(1 / 6)$ \\
\hline
\end{tabular}

-: Normal, +: Mild, ++: Moderate, +++: Severe

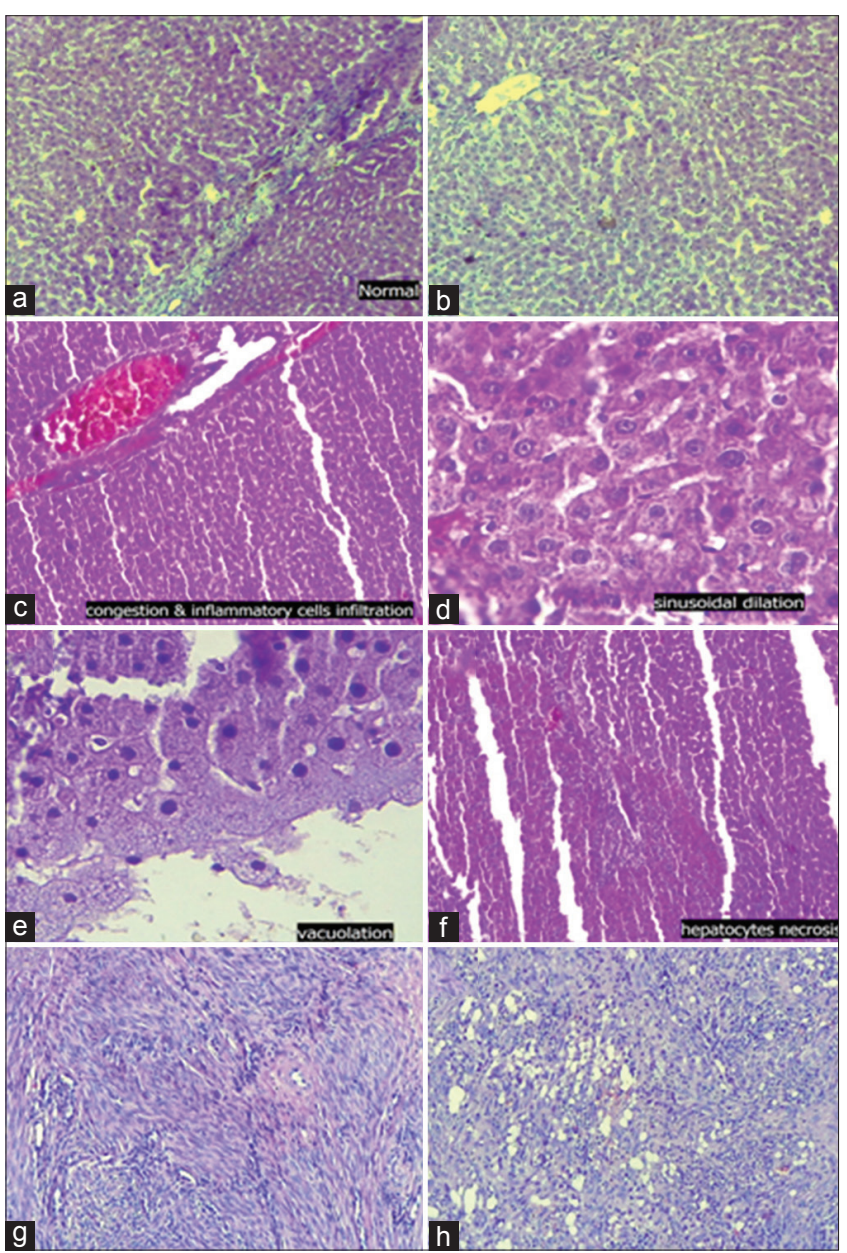

Fig. 3: Effect of zileuton and MK-886 treatments on liver histopathology. (a) negative control, (b) vehicle group, (c-f): Dx-treated group, (g) zileuton-treated group, (f) Mk-886treated group

\section{DISCUSSION}

Dx is a drug used for the treatment of cancer, especially leukemia and solid tumors. Therapy by Dx has limited toxicity as compared with quinone anthracyclines [21]. The free radical formation was thought that it was produced by Dx administration [22]. The hepatotoxicity is occurring by treatment with Dx in many animals [23]. The significant steatosis, the vascular damage, and focal damage in the liver cell are appearing after Dx administration [24]. $20 \mathrm{mg} / \mathrm{kg}$ of Dx is increased ALAT level in serum double normal in mice [25]. These results are the same in our study. However, there are several changes in enzyme level (ASAT and ALAT) after injection of DX dose alone. DX can increase the levels of ASAT and ALAT in serum and that agree with another study [26]. Shiyun Pu shows pharmacological inhibition or deletion of 5-LO prevented paracetamol increased serum ALAT and ASAT values and hepatic necrosis [27]. Gul Daglar used the measurement of the ALAT concentration as a marker for the detection of parenchymal cell injury of the liver. The ALAT levels increase significantly after injection. Furthermore, MK-886 treatment increases in ALAT concentration in hepatic I/R when compared with I/R group, decreasing of the ALAT concentration is an indicator of liver protection against hepatic $I / R$ injury [28].

Our results demonstrated that MK- and zileuton-treated groups cause higher GSH levels and lower MDA levels when compared with Dx-treated group. Several local types of research demonstrated that zileuton associated with higher GSH and lower MDA levels when compared with the induced group $[14,15]$. Tu et al. found that zileuton causes decreasing MDA level in rats. The selective 5-LOX inhibitor, zileuton, inhibited NF- $\mathrm{\kappa B}$ activation, and 12 reduced the activation and expression of iNOS. Furthermore, NF- $\kappa B$ can regulate iNOS expression and some inflammatory mediators [29]. No supported data are available for the effect of Mk-886 on oxidative markers.

Furthermore, our study showed that those treated with zileuton plus Dx liver tissues showed vacuolation, inflammatory cell infiltration, and sinusoidal dilation with mild congestion, while those treated with Mk-886 plus Dx showed nearly normal liver pathophysiology. In highfat-diet-induced non-alcoholic fatty liver disease (NAFLD), Kuifen concluded that inhibitor zileuton and inhibition of 5-lipoxygenase progression model and so AA/5-LOX pathway stimuli the progression of NAFLD, which could reverse by zileuton administration [30].

\section{CONCLUSION}

Through our finding, we can conclude that Zileuton and MK-886 have a protective effect against hepatic damage induced by doxorubicin.

\section{AUTHORS' CONTRIBUTIONS}

All authors contribute equally in the preparation of this work.

\section{CONFLICTS OF INTEREST}

The author declares no conflicts of interest.

\section{REFERENCES}

1. Zordoky BN, Mohamed AA, Aboutabl ME, El-Kadi AO. Acute doxorubicin toxicity differentially alters cytochrome P450 expression andarachidonic acid metabolism in rat kidney and liver. Am Soc Pharm Exp Ther 2011;39:1440-50.

2. Swift LP, Cutts SM, Rephaeli A, Nudelman A, Phillips DR. Activation of adriamycin by the $\mathrm{pH}$-dependent formaldehyde-releasing prodrug 
hexamethylenetetramine. Mol Cancer Ther 2003;2:189-98.

3. Sbitti EY, Ichou M, Errihani H. The role of chemotherapy in the treatment of Kaposi's sarcoma. J Cancer Sci Ther 2011;3:145-8.

4. Kaur A, Kumar MK. Doxorubicin: A critical review on toxicity. J Pharm Res 2012;5:2890-4.

5. Injac R, Strukelj B. Recent advances in protection against doxorubicininduced toxicity. Technol Cancer Res Treat 2008;7:497-516.

6. Bulucu F, Ocal R, Karadurmus N, Sahin M, Kenar L, Aydin A, et al. Effects of N-acetylcysteine, deferoxamine and selenium on doxorubicininduced hepatotoxicity. Biol Trace Elem Res 2009;132:184-96.

7. Rašković A, Stilinović N, Kolarović J, Vasović V, Vukmirović S, Mikov M, et al. The protective effects of silymarin against doxorubicin-induced cardiotoxicity and hepatotoxicity in rats. Molecules 2011;16:8601-13.

8. Indu R, Azhar TS, Nair A, Nair CK. Amelioration of doxorubicin induced cardio-and hepato-toxicity by carotenoids. J Cancer Res Ther 2014;10:62-7.

9. Heide RS, L'Ecuyer TJ. Molecular basis of anthracycline-induced cardiotoxicity. Heart Metab 2007;35:1-4.

10. El-Sayyad HI, Ismail MF, Shalaby FM, Abou-El-Magd RF, Gaur RL, Fernando A, et al. Histopathological effects of cisplatin, doxorubicin and 5-flurouracil (5-FU) on the liver of male albino rats. Int J Biol Sci 2009;5:466-73.

11. Gunda S, Kour SM. Evaluation of hepatoprotective effect of morin against doxorubicin-induced hepatotoxicity in Wistar rats. Eur J Biomed Pharm Sci 2018;5:663-8.

12. Mezher MN, Dakhil AS, Abdul-Jawad DH. Role of epstein-barr virus (EBV) in human females with breast cancer. J Pharm Sci Res 2017;9:1173-7

13. Peters-Golden M, Henderson WR Jr. Leukotrienes. N Engl J Med 2007;357:1841-54

14. Hadi NR, Mohammad BI, Ajeena IM, Sultan AM, Majeed S, Hussain T, et al. Leukotriene synthesis inhibitors modulate atherosclerosis progression in hypercholesterolemic rabbits. Res J Pharm Biol Chem Sci 2015;6:362-70.

15. Hadi NR, Mohammad BI, Almudhafer A, Yousif N, Sultan AM. Montelukast and zileuton retard the progression of atherosclerosis via down-regulation of the inflammatory and oxidative pathways. J Clin Exp Cardiolog 2013;4:250

16. Dakhil AS. Association of serum concentrations of proinflammatory cytokines and hematological parameters in rheumatoid arthritis patients. J Pharm Sci Res 2017;9:1966-74.

17. Mansour MA, El-Kashef HA, Al-Shabanah OA. Effect of captopril on doxorubicin-induced nephrotoxicity in normal rats. Pharmacol Res 1999;39:233-7.

18. Rossi A, Pergola C, Koeberle A, Hoffmann M, Dehm F, Bramanti P, et al. The 5-lipoxygenase inhibitor, zileuton, suppresses prostaglandin biosynthesis by inhibition of arachidonic acid release in macrophages. Br J Pharmacol 2010;161:555-70.

19. Dakhil AS. Biosynthesis of silver nanoparticle (AgNPs) using Lactobacillus and their effects on oxidative stress biomarkers in rats. J King Saud Univ Sci 2017;29:462-7.

20. Lee IC, Kim SH, Baek HS, Moon C, Bae CS, Kim SH, et al. Melatonin improves adriamycin-induced hepatic oxidativedamage in rats. Mol Cell Toxicol 2013:9:257-65.

21. Jamieson D, Boddy AV. Pharmacogenetics of genes across the doxorubicin pathway. Expert Opin Drug Metab Toxicol 2011;7:1201-10.

22. Llesuy SF, Arnaiz SL. Hepatotoxicity of mitoxantrone and doxorubicin. Toxicology 1990;63:187-98.

23. Dakhil AS, Al-Hajjiah NN, Shlash RF. Identification of factor viii gene mutations in patients with haemophilia A. Int J Res Pharm Sci 2018;9:274-28.

24. Pedrycz A, Wieczorski M, Czerny K. The influence of a single dose of Adriamycin on the pregnant rat female liver-histological and histochemical evaluation. Ann Univ Mariae Curie Sklodowska Med 2004:59:319-23.

25. Miranda CJ, Makui H, Soares RJ, Bilodeau M, Mui J, Vali H, et al. Hfe deficiency increases susceptibility to cardiotoxicity and exacerbates changes in iron metabolism induced by doxorubicin. Blood 2003;102:2574-80

26. Mohamad RH, El-Bastawesy AM, Zekry ZK, Al-Mehdar HA, AlSaid MG, Aly SS, et al. The role of curcuma longa against doxorubicin (Adriamycin)-induced toxicity in rats. J Med Food 2009;12:394-402.

27. Al-Hajjiah NN, Almkhadree MA. The effect of maternal anemia on the anthropometric measurements in full-term neonates. Asian J Pharm Clin Res 2018;11:3680-1.

28. Al-Hajjiah NN, Al-Shamsi MM, Al-Shami MM. The rate of parental refusal lumbar puncture in the maternity and children teaching hospital in Diwaniyah, Iraq. J Pharm Sci Res 2018;10:2680-1.

29. Tu XK, Yang WZ, Wang CH, Shi SS, Zhang YL, Chen CM, et al. Zileuton reduces inflammatory reaction and brain damage following permanent cerebral ischemia in rats. Inflammation 2010;33:344-52.

30. Ma K, Chen Y, Liang X, Miao J, Zhao Q. Inhibition of 5-lipoxygenase inhibitor zileuton in high-fat diet-induced nonalcoholic fatty liver disease progression model. Iran J Basic Med Sci 2017;20:1207-12. 